\title{
Halftoning by Error Diffusion Method in Visual Cryptography for Greyscale Image
}

\author{
Asad Mansoori \\ Computer Science Engineering, \\ IFTM University \\ Moradabad, \\ U.P, India
}

\author{
Vijay Kumar Joshi \\ Assistant Professor, \\ Department Of \\ Computer Science Engineering, \\ IFTM UNIVERSITY, \\ Moradabad, U.P, India
}

\begin{abstract}
Visual Cryptography (VC) is a technique used for protecting image based secrets. The main concept of the original visual cryptography scheme is to encrypt a secret image into some shares. Secret information cannot be revealed with few shares. All shares are necessary to combine to reveal the secret image. Halftoning is the important feature of visual cryptography. It provides security at the early stage of visual cryptography. Halftoning is the reprographic techniques, whose process of converting large tone (high intensity value of pixel) image to low tone (low intensity value of pixel) image.

The visual cryptography is a hide information technology. The visual cryptography uses sharing method apply for hide information. Here used the $(2,2)$ greyscale image method, 2 shares out of 2 stack the secret will reveal and less than 2 shares are not work. Here, proposed a modified algorithm of error-diffusion half toning in visual cryptography. Increment the quality of image by modified method according to different parameters. According to modified method, error distributed to nearest pixel. The compared author method with Floyed, Jarvis, and Stucki throw PSNR and UQI parameters.

The visual secret scheme decrypts the secret image using Human Visual System (HVS) without any computation. Here, used to $(2,2)$ generate visual sharing system for halftone gray scale image. Here generate shares of halftone image and recovered secret image.
\end{abstract}

\section{Keywords}

Visual Cryptography, Halftone, Error-Diffusion, Shares, PSNR, UQI

\section{INTRODUCTION}

This technique first Naor and Shamir Sun 1994 invented in which they have two transparent picture exercised including a photo exhibit to another side. The visual cryptography to implement hide a transparent sheet to the print easiest way. There are always the same number white (transparent) and black blocks. A Pixel is divided into two parts, there are white and black block. The visual cryptography uses sharing method apply for hide information. In this case use $(k, n)$ greyscale image method, $\mathrm{k}$ shares out of $\mathrm{n}$ stack the secret will reveal and less than $\mathrm{k}$ shares are not work. The messages transfer one to other place the cryptography provide security using by key on internet. Cryptography any massage brake in more than two part according to security system. Every part move to different-different root on network. The visual cryptography according to security any image make two and more than two share. Original image information divided by equal in all share. The single share image information can't see human seye. When all share can't be overlapping till then see no fulfil original information.Human vision technology works on a certain parameter.

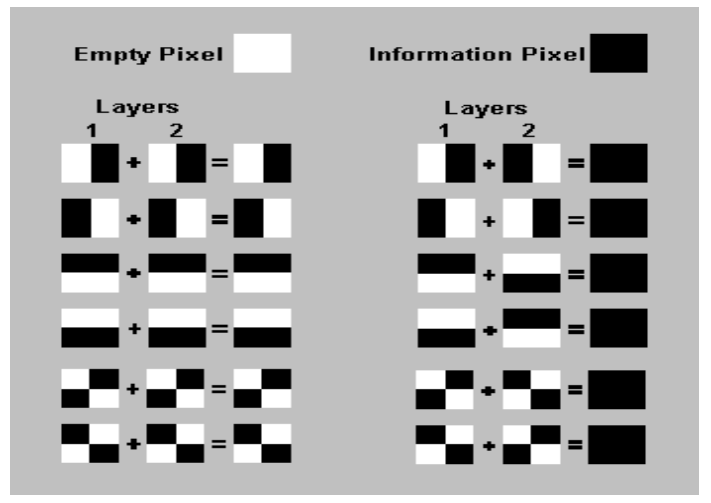

Figure-1 Construction of $(2,2)$ VC Scheme

\section{ERROR-DIFFUSION}

The halftone any image pixel value camper to taken threshold value. The simplest method of threshold each pixel of the image changed to black pixel with the image intensity value I $(i, j)$, which is its real value than the fixed constant $\mathbf{T}(i, j)$ fi I $(i, j)<T(i, j)$ is less than the black pixel more so if the white pixel assume. The technique is use to change the image as dotes that is only imaginary fiction. It is estimated that the pixel is part of the actual image. He is a kind of protective layer generated image of the image is formed in part in the creation of this patterns of a particular type used. The halftone colauther printed process with a colauther ink. The printing model is dimension spacing model reduces of the dots in the image. A fundamental reproductive process Combine small partial dots human eyes is displayed on the lower tones. Round dots is used in most public. Where the dots are used to display various types of dots each have their own specialties and all of them together to avoid unwanted effects, general, printing method is depends on the size.

The thresholding block where the output $\mathrm{g}(\mathrm{m}, \mathrm{n})$ -

$$
\mathbf{g}(\mathbf{m}, \mathbf{n})=\left\{\begin{array}{c}
\mathbf{1}, \text { if } \mathbf{d}(\mathbf{m}, \mathbf{n})>t(m, n) \\
\text { 0, otherwise }
\end{array}\right\}
$$

The $\mathrm{t}(\mathrm{m}, \mathrm{n})$ is a threshold value $\mathrm{h}(\mathrm{m}, \mathrm{n})$ is an error filtering and $e(m, n)$ input between $d(m, n)$ and $g(m, n)$ and $f(m, n)$ is represent the $(m, n)^{\text {th }}$ pixel value of the input grayscale image The calculate $d(m, n)$ value

$$
d(m, n)=f(m, n)-\sum h(k, l) \text { e }(m-k, n-l)
$$




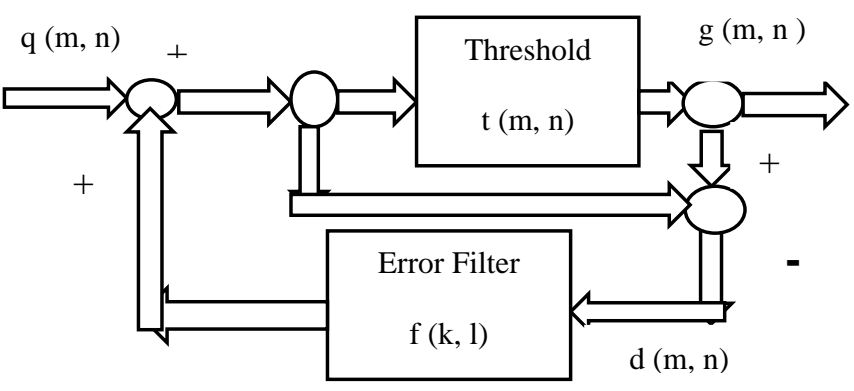

Figure-2 Block diagram of Error-Diffusion

Error diffusing which came on an image pixel is different. The actual values of the pixel on him. We have delivered real value of the pixel after the halftone is received. There is without any errors. If the error diffusion is error in an image in circumstances. The error is distributing on its surrounding pixels. Halftone image of each pixel value is between 0 and 1 because of an image after the halftone image pixel display is only binary image. The threshold value above the value of 0 and 1 as the value of the pixel to fully bring the error of the pixels on implanted with the error diffusion method.

\section{LITERATURE REVIEW}

C.C Chang, and W.L. Tai[1] develop a method to hide the secret halftone image into two camouflaged halftone images. When the two camouflaged halftone images are stacked together, the secret halftone image can be revealed by the human eye. The halftone images, which have gone through error diffusion, are used to do image hiding. If there exist areas with white dots in the corresponding positions of two halftone images, the secret pattern in the overlaying area will be destroyed. We adjust the range of pixel values such that there is no area with exclusively white pixels or black pixels in two halftone images.

DONG Lin, WANG Dao Shun, LI et al [2] represent theory based on $(2, n)$ secret sharing scheme ( 2 out of $n$ ) improve the contrast propose for binary image. In this paper increase the gray scale image improved to the colautherset in black/white image increase the pixel stored colautherand apply bit level processing and Boolean operation perform (XOR and OR operation). The light of pixel controls the electric current in computer screen. The general printer can only control a single pixel to be printed (black pixel) or not to be printed (white pixel).

Pradeep Kumar Sharma and Hari Mohan Singh [3] introduced the new method used for visual cryptography, used the first time plaintext attach the key the same key used decrepit the original form This work has illustrated that the pixel intensity division method could be used to effective to improve the performance of an image for transmitting any image through E-mail or through any other medium. In order to reduce the more computation work and improve the quality of image with security a two level authentication scheme has been proposed to avoid different security attacks and threats.

Zhou, Zhi, Gonzalo R. Arce [4] are Halftone visual cryptography and Yang, Ching-Nung, Pin-Wei Chen [5] are Aspect ratio invariant visual cryptography by image filtering and resizing. Applying the rich theory of blue noise half toning into the construction mechanism of conventional VC, used in a number of visual secret sharing applications which require high-quality visual images. The recovered image obtained using proposed error diffusion filter are much better than existing error filters and proposed error filter gives maximum values.

Aman Kamboj and D.K.Gupta [6] introduced the half toning methods described in previous section shows the method error are distributed in both forward and backward direction. A halftone image $\mathrm{I}$ is obtained by applying error diffusion halftone method on a grey scale image G. The method scan image one row and one pixel at a time. The generated pixel is either full black or full white. Shares are generated using basic visual $(2,2)$ scheme. A secret pixel p into a $\mathrm{Q} 1{ }^{*} \mathrm{Q} 2$ halftone cell in each of the two shares, only two pixels, referred to as the secret information pixels, in each halftone cell need to be modified. The two secret information pixels should be at the same positions in two shares quality depending on their own opinion. The objective evaluation uses automatic algorithms to assess the quality of the image without human interfere.

According to El-Tigani B. Abdelsatir, Sahar Salahaldeen, Hyam Omar and Afra Hashim [7] used method secret sharing threshold scheme and as a result we propose a new scheme for secret image sharing from quadratic residues and incorporated with Shamir secret sharing method in terms of flexibility in the number of secret shares which is limited to only two shares. The proposed scheme shows great performance in terms of low distortion in the reconstructed image.

Abhishak Kumar Mishra and Ashutosh Gupta [8] used to transforming grayscale level image into a bi level image in halftone and the applied the visual cryptography method directly halftone image. The $[n, n]$ visual cryptography based sharer encryption method .The grayscale of pixel halftone technic to transform the original image convert binary image 0 's and 1's consist. The each pixel divided into fauthersub pixel. This method applied [2*2] matrix and five different gray level can be represent by the set of matrices in an image the select the 15 pixel in image. When the pixel value is 128 .

Kang, Gonzalo R. Arce, and Heung-Kyu Lee [9] approach on color extended visual cryptography for General access structures using the principle of Error diffusion This method uses a two-phased encryption algorithm of (extended visual cryptography scheme) EVCS for GASs. The first phase of Error Diffusion Half toning algorithm is used to generate color shares for color secret image. Half toning is a binarization technique that converts gray scale image into a binary image. It converts gray scale pixel into binary using a threshold value Error diffusion will generates halftone shares with high quality.

Ram Krishna Jha and Abhijeet Mustafa [10] his research on visual cryptography $(\mathrm{k}, \mathrm{n}) \mathrm{k}$ in which the threshold value of shares presented is considered Genetic Algorithm with 2-outof-2 to an image generated on the basis of the He later 3-outof- 4 pixels of an image using matrix $(3,4)$ based on shared sub-pixels in a pixel is divided. He changed the image of the binary image within the range of 3-out-of- 4 cooperation XOR method to hide the original image information can also change the size of the actual image.

Sandeep Katta [11] stock for the image pixel gray scale image at least to change their colautherother than the colautherof the pixel is made up of. He's 2-out of-3 to the image that has the possibility to base value. The normally two binary image for which to add or decrease the OR operation is used. Visual Secret Sharing Scheme using Grayscale Images using minimum pixel expansion have been proposed for black and white (binary) secret images with the number of probabilistic VSS schemes. In case binary image is divided into two share 
and after that all sharing binary image pixel value addition after that same value comparing original image pixel without any lose.

\section{PROPOSED METHOD}

Visual cryptography information inside the image to try to keep more secure being. This technology can turn any image into halftone image has errors in it can be overcome through error-Diffusion of the image pixels as Chase board displays. Author use to theology gray scale image because it represents only two colors, black and white only. Author image is a gray scale image in which $m * n$ matrix of $m$ row and $n$ column displays. Threshold limit values and the corresponding boundaries of author research, we have to reduce. The pixel value of each pixel of the image between 0 and 1 are lower or higher than the value of a pixel.

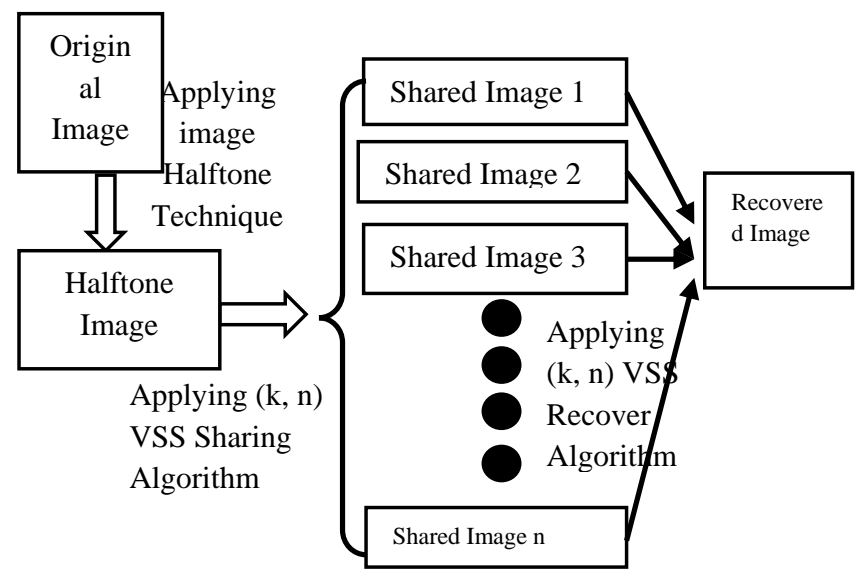

Figure-3 Block Diagram Process Work

We have a $512 * 512$ pixel image and 127.5 threshold value uses the image of the old value by removing the assumption that the value we convert image to double precision threshold value, then the threshold value of double precision image Divided we do. Made for a random variable vector a scalar of observations not.

Floyd, Jarvis and Stucki algorithms apply Lena $512 * 512$ pixel gray scale image changed Halftone image through errorDiffusion method the threshold value is 127.5 considered same parameter to gray scale image

\subsection{Floyd-Steinberg dithering Method}

Then 16 on a pixel error is error to those who have the proportions $7 / 16,3 / 16,5 / 16,1 / 16$ pixels which are split on the error, according to these proportions is distributing the nearby pixels. The method used to deliver to him the sum is 1 to 4 pixels. The result of this algorithm is rather impressive even compared to the best ordered dither results we could achieve:

Table-1 Filter coefficients used by Floyd and Steinberg

\begin{tabular}{|c|c|c|}
\hline & $\times$ & $\frac{7}{16}$ \\
\hline$\frac{3}{16}$ & $\frac{5}{16}$ & $\frac{1}{16}$ \\
\hline
\end{tabular}

Volume 148 - No.10, August 2016

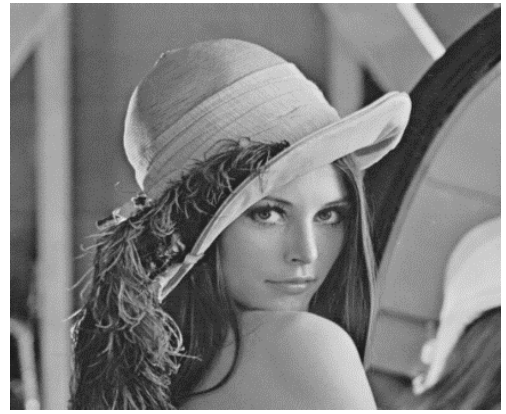

Figure-(A)

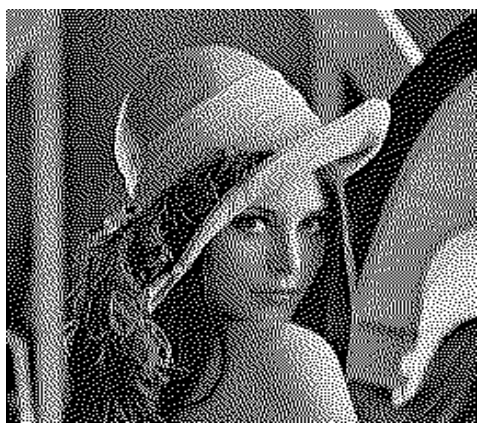

Figure-(B)

Figure-4 (A) Lina Original Image, (B) Halftone Image by Floyd

\subsection{Jarvis, Judice and Ninke dithering}

Then 16 on a pixel error is error to those who have the proportions $7 / 48,5 / 48,5 / 48,1 / 16,7 / 48,5 / 48,1 / 16,1 / 48$, $1 / 16,5 / 48,1 / 16,1 / 48$ pixels which are split on the error, according to these proportions is distributing the nearby pixels.

Table-2 Filter weight used by Jarvis, Judice and Ninke

\begin{tabular}{|c|c|c|c|c|}
\hline & & $\times$ & $\frac{7}{48}$ & $\frac{5}{48}$ \\
\hline$\frac{1}{16}$ & $\frac{5}{48}$ & $\frac{7}{48}$ & $\frac{5}{48}$ & $\frac{1}{16}$ \\
\hline$\frac{1}{48}$ & $\frac{1}{16}$ & $\frac{5}{48}$ & $\frac{1}{16}$ & $\frac{1}{48}$ \\
\hline
\end{tabular}

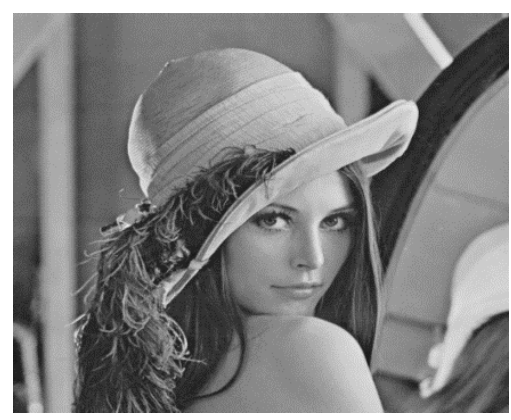

Figure- (A) 


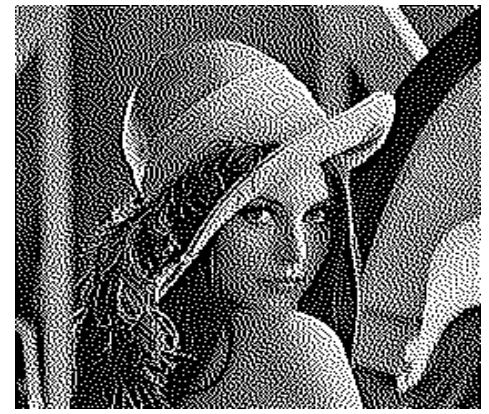

Figure- (B)

Figure-5 (A) Lina Original Image, (B) Halftone Image by Jarvis

\subsection{Stucki dithering}

According to Stucki that he Lena $512 * 512$ pixel gray scale image changed Halftone image through error-Diffusion method the threshold value is 127.5 considered to gray scale image. By then 16 on a pixel error is error to those who have the proportions $4 / 21,2 / 21,1 / 21,2 / 21,4 / 21,2 / 21,1 / 21,1 / 42$, $1 / 21,2 / 21,1 / 21,1 / 42$ pixels which are split on the error, according to these proportions is distributing the nearby pixels.

Table-3 Filter weight used by Stucki.

\begin{tabular}{|c|c|c|c|c|}
\hline & & $\times$ & $\frac{4}{21}$ & $\frac{2}{21}$ \\
\hline$\frac{1}{21}$ & $\frac{2}{21}$ & $\frac{4}{21}$ & $\frac{2}{21}$ & $\frac{1}{21}$ \\
\hline$\frac{1}{42}$ & $\frac{1}{21}$ & $\frac{2}{21}$ & $\frac{1}{21}$ & $\frac{1}{42}$ \\
\hline
\end{tabular}

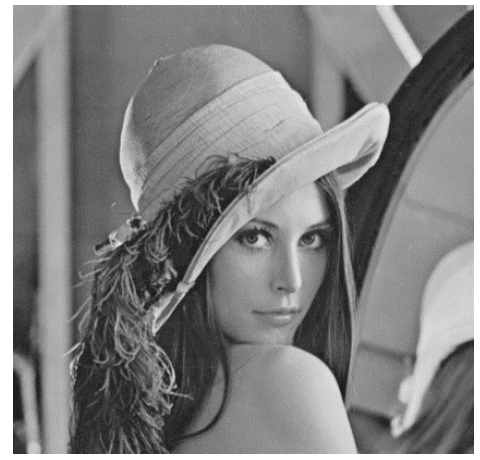

Figure-(A)

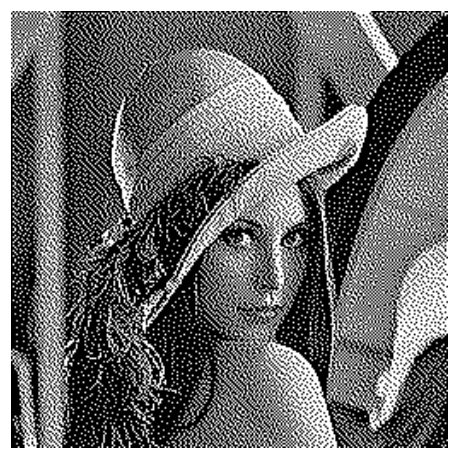

Figure- (B)

Figure-6 (A) Lina Original Image, (B) Halftone Image by Stucki
International Journal of Computer Applications (0975 - 8887)

Volume 148 - No.10, August 2016

\subsection{Proposed Method}

Error diffusion in the method from time to time many researchers have presented their own draft, but these three as the base for any new method created and their results are evaluated.

The grayscale image in a $512 * 512$ unit 8 bit image. The range of image pixel 0 to 255 this is a pixel intensity value, grayscale image apply the threshold value 127.5 apply to the after that 127.5 less pixel value as a 0 and grater then 1.The image value in 0 and 1 form represent. We also distribute the error to him in more and more parts of the pixel is not delivered to the error. Gray scale image halftone after changing it, came the error to correct auther 200 run the error by distributing the error of 49 pixels distributed on the turn and the process until moving until the entire error does not solve said.

\section{Algorithm Halftone Process}

1. Initialize Variable

I = Input Image;

$[\mathrm{M}, \mathrm{N}]=$ Size (inImage); Where $\mathrm{M}=$ row and $\mathrm{N}=$ column

2. For $i=1$ to $M, j=1$ to $N$

3. $\mathrm{T}=127.5$; Where $\mathrm{T}=$ Threshold Value

If

$H(i, j)=I(i, j) \geq T$; Where H=Halftone Image

Set $I(i, j)=1$

else

Set $I(i, j)=0$

4. Error $=I(i, j)-H(i, j)$

5. For

$\mathrm{i}=1$ to $\mathrm{M}-5, \mathrm{j}=1$ to $\mathrm{N}-5, \mathrm{i}++, \mathrm{j}++$

6. Distribute error $\mathrm{h}(0,1)=8 / 200, \mathrm{~h}(0,2)=7 / 200, \mathrm{~h}(0,3)$ $=6 / 200, \mathrm{~h}(0,4)=5 / 200, \mathrm{~h}(0,5)=4 / 200$ to the right pixel in image

7. Distribute error h $(1,0)=8 / 200$, h $(2,0)=7 / 200$, h $(3,0)$ $=6 / 200, \mathrm{~h}(4,0)=5 / 200$ to the bottom pixel in image

8. Distribute error h $(1,-1)=7 / 200, \mathrm{~h}(1,-2)=6 / 200, \mathrm{~h}(1,-3)$ $=5 / 200, \mathrm{~h}(1,-4)=4 / 200, \mathrm{~h}(1,-5)=3 / 200, \mathrm{~h}(2,-1)=6 / 200, \mathrm{~h}$ $(2,-2)=5 / 200, \mathrm{~h}(2,-3)=4 / 200, \mathrm{~h}(2,-4)=3 / 200, \mathrm{~h}(2,-5)$ $=2 / 200, \mathrm{~h}(3,-1)=5 / 200, \mathrm{~h}(3,-2)=4 / 200, \mathrm{~h}(3,-3)=3 / 200, \mathrm{~h}$ $(3,-4)=2 / 200$, h $(3,-5)=1 / 200, \mathrm{~h}(4,-1)=4 / 200$, h $(4,-2)$ $=3 / 200, \mathrm{~h}(4,-3)=2 / 200, \mathrm{~h}(4,-4)=1 / 200, \mathrm{~h}(4,-5)=1 / 100$, to the left diagonal pixel in image

9. Distribute error h $(1,1)=7 / 200, \mathrm{~h}(1,2)=6 / 200$, h $(1,3)$ $=5 / 200, \mathrm{~h}(1,4)=4 / 200, \mathrm{~h}(1,5)=3 / 200, \mathrm{~h}(2,1)=6 / 200, \mathrm{~h}(2$, 2) $=5 / 200, \mathrm{~h}(2,3)=4 / 200, \mathrm{~h}(2,4)=3 / 200, \mathrm{~h}(2,5)=2 / 200, \mathrm{~h}$ $(3,1)=5 / 200, \mathrm{~h}(3,2)=4 / 200, \mathrm{~h}(3,3)=3 / 200, \mathrm{~h}(3,4)=2 / 200$, h $(3,5)=1 / 200$,h $(4,1)=4 / 200$, h $(4,2)=3 / 200$, h $(4,3)$ $=2 / 200, \mathrm{~h}(4,4)=1 / 200, \mathrm{~h}(4,5)=1 / 100$, to the right diagonal pixel in image. 
Table-4 Filter weight used by Proposed Method

\begin{tabular}{|c|c|c|c|c|c|c|c|c|c|c|}
\hline & & & & & $*$ & 8 & 7 & 6 & 5 & 4 \\
\hline & & & & & & 200 & $\overline{200}$ & $\overline{200}$ & 200 & 200 \\
\hline 3 & 4 & 5 & 6 & 7 & 8 & 7 & 6 & 5 & 4 & 3 \\
\hline$\overline{200}$ & 200 & 200 & 200 & 200 & 200 & 200 & 200 & 200 & 200 & 200 \\
\hline 2 & 3 & 4 & 5 & 6 & 7 & 6 & 5 & 4 & 3 & 2 \\
\hline$\overline{200}$ & 200 & 200 & 200 & 200 & 200 & 200 & 200 & 200 & 200 & 200 \\
\hline 1 & 2 & 3 & 4 & 5 & 6 & 5 & 4 & 3 & 2 & 1 \\
\hline$\overline{200}$ & 200 & 200 & 200 & 200 & 200 & $\overline{200}$ & $\overline{200}$ & $\overline{200}$ & 200 & 200 \\
\hline 1 & 1 & 2 & 3 & 4 & 5 & 4 & 3 & 2 & 1 & 1 \\
\hline$\overline{00}$ & 00 & 200 & 200 & 200 & 200 & 200 & $\overline{200}$ & 200 & 200 & $\overline{100}$ \\
\hline
\end{tabular}

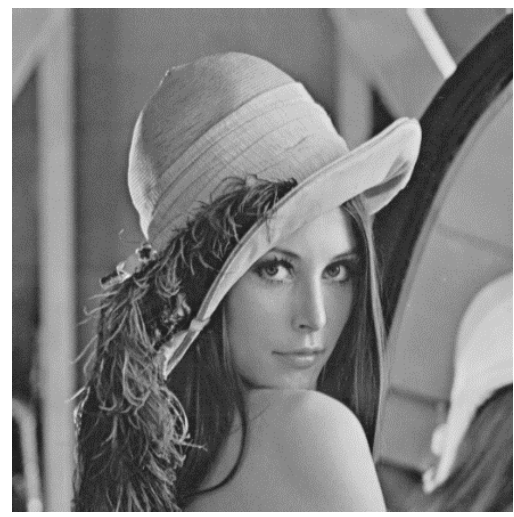

Figure-(A)

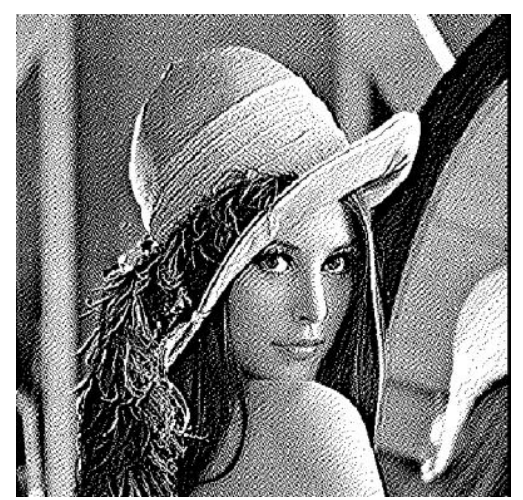

Figure-(B)

Figure-7 (A) Lina Original Image, (B) Halftone Image by Proposed Method

PSNR: The simplest and most widely used pixel wise error based measures are mean squared error (MSE) and peak signal-to-noise ratio (PSNR). The MSE is the squared intensity differences between the reference and the test image pixels, which defined by:

$$
\begin{gathered}
{\left[\mathrm{MSE}=\frac{1}{\mathrm{MN}} \sum_{m=1}^{M} \sum_{n=1}^{N}\left[f(m, n)-f^{\prime}(m, n)\right]^{2}\right]} \\
{\left[P S N R=10 \log _{10} \frac{255^{2}}{M S E}\right]}
\end{gathered}
$$

Where $f(m, n)$ is the original image and $f^{\prime}(m, n)$ is the halftone image.

Universal Image Quality Index (UQI); Let X and Y be the original and test image signal respectively where
International Journal of Computer Applications (0975 - 8887) Volume 148 - No.10, August 2016

$$
\mathbf{X}=\left\{\mathbf{x}_{\mathbf{i}} \mid \mathbf{i}=\mathbf{1}, \mathbf{2} \ldots \mathbf{N}\right\} \mathbf{Y}=\left\{\mathbf{y}_{\mathbf{i}} \mid \mathbf{i}=1,2 \ldots \mathbf{N}\right\}
$$

$$
\left[\mathrm{UQI}=\frac{4 \sigma_{\mathrm{XX}} \overline{\mathrm{xy}}}{\left(\overline{\mathrm{x}}^{2}+\overline{\mathrm{y}}^{2}\right)\left(\sigma_{\mathrm{x}}^{2}+\sigma_{\mathrm{y}}^{2}\right)}\right]
$$

Where

$$
\bar{x}=\frac{1}{N} \sum_{i=1}^{N} x_{i} \quad \bar{y}=\frac{1}{N} \sum_{i=1}^{N} y_{i}
$$

The 2 Out-of 2 method in which Sharing image pixels is changing the value of 0 and 1 in binary form and $2 \times 2$ matrix of the image by changing the matrix is divided into two parts of the overlapping them when we can get the real image of the Information. When we column image and the value of 0 and 1

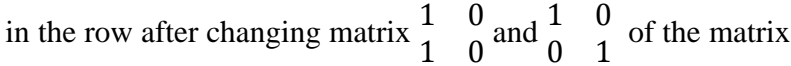
as compared to white and black respectively displays.

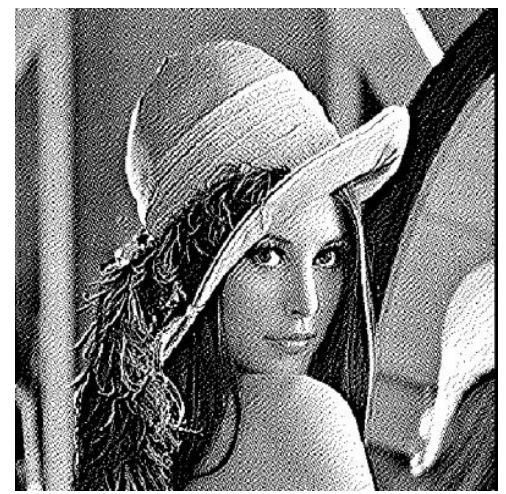

Figure-(A)

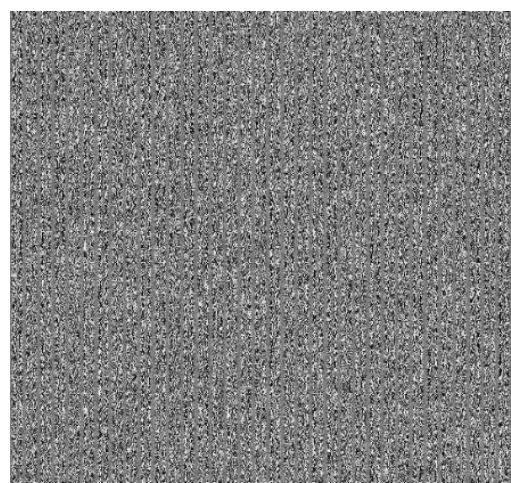

Figure- (B)

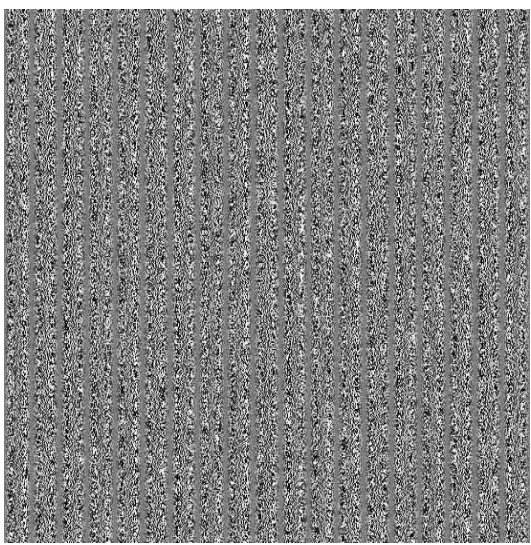

Figure-(C) 


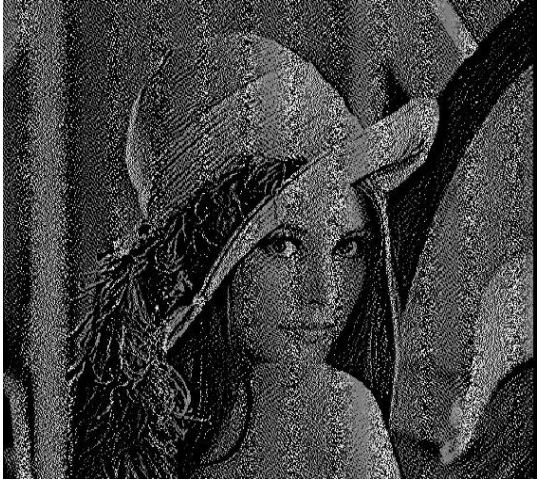

Figure-(D)

Figure-8 (A) Lina Halftone Image by Proposed Method, (B) Share1, (C) Share2, (D) Recover Image

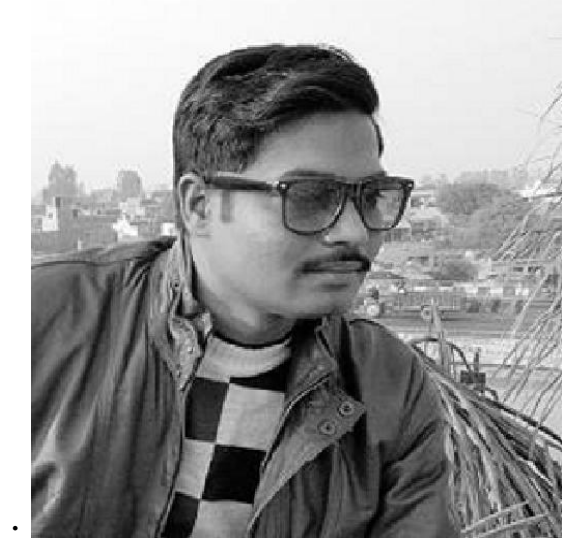

Figure-(A)

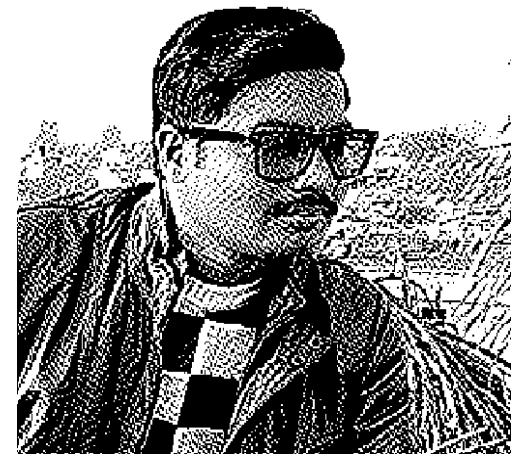

Figure-(B)

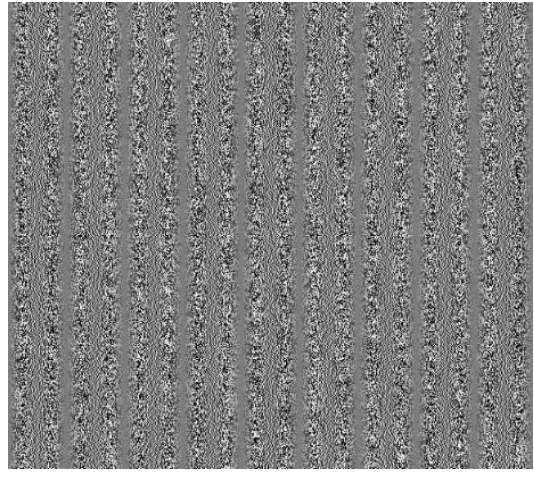

Figure-(C)
International Journal of Computer Applications (0975 - 8887) Volume 148 - No.10, August 2016

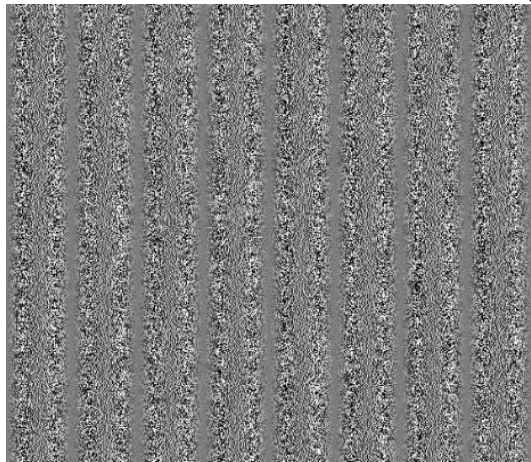

Figure-(D)

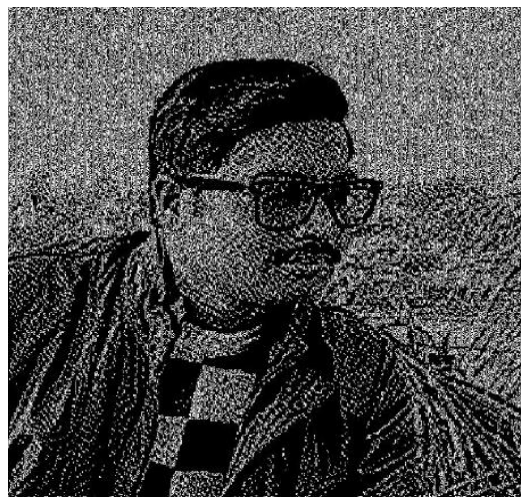

Figure-(E)

Figure-9 (A) Author Original Image, (B) Halftone Image by Proposed Method (C) Share1,(D)Share2, (E) Recover Image

\subsection{Result Analysis}

Table-5 PSNR and UQI Image Quality for Lina Image

\begin{tabular}{|c|c|c|}
\hline $\begin{array}{c}\text { Type Of } \\
\text { Algorithm }\end{array}$ & PSNR & UQI \\
\hline Floyed & 5.7552 & $8.3620 \mathrm{e}-05$ \\
\hline Jarvis & 5.7554 & $1.5300 \mathrm{e}-04$ \\
\hline Stucki & 5.7553 & $1.2576 \mathrm{e}-04$ \\
\hline $\begin{array}{c}\text { Proposed } \\
\text { Method }\end{array}$ & 5.7566 & $2.6346 \mathrm{e}-04$ \\
\hline
\end{tabular}

Table-6 PSNR and UQI Image Quality for author Image

\begin{tabular}{|c|c|c|}
\hline $\begin{array}{c}\text { Type Of } \\
\text { Algorithm }\end{array}$ & PSNR & UQI \\
\hline Floyed & 3.9680 & $1.6657 \mathrm{e}-04$ \\
\hline Jarvis & 3.9682 & $2.2744 \mathrm{e}-04$ \\
\hline Stucki & 3.9682 & $2.1063 \mathrm{e}-04$ \\
\hline $\begin{array}{c}\text { Proposed } \\
\text { Method }\end{array}$ & 3.9690 & $4.5295 \mathrm{e}-04$ \\
\hline
\end{tabular}




\section{CONCLUSION}

In this paper, the author proposed a modified algorithm of error-diffusion halftoning in visual cryptography and also compared our method with Floyed, Jarvis, and Stucki by the PSNR and UQI parameters. The modified algorithm method is batter from Floyed, Jarvis, and Stucki according to the value of PSNR and UQI parameters, after that generate share of halftone image and recovered image.

In future, improve to the quality of halftone image with PSNR and UQI as well as another parameters and provide a high security and quality of image in other visual cryptography schemes for grayscale image.

\section{REFERENCES}

[1] C. Chang, C. Chan and W. Tai, "Hiding a halftone secret image in two camouflaged halftone images", Pattern Recognition and Image Analysis, vol. 16, no. 3, pp. 486496, 2006.

[2] L. Dong, D. Wang, S. Li and Y. Dai, "(2,n) secret sharing scheme for gray and color images based on Boolean operation", Science China Information Sciences, vol. 55, no. 5, pp. 1151-1161, 2011.

[3] P. Sharma and H. Singh, "Visual Cryptography Scheme for Gray Scale Images based on Intensity Division", International Journal of Current Engineering and Technology, vol. 4, no. 1, pp. 225-230, 2014.

[4] Zhi Zhou, G. Arce and G. Di Crescenzo, "Halftone visual cryptography", IEEE Transactions on Image Processing, vol. 15, no. 8, pp. 2441-2453, 2006.
International Journal of Computer Applications (0975 - 8887)

Volume 148 - No.10, August 2016

[5] C. Yang, P. Chen, H. Shih and C. Kim, "Aspect ratio invariant visual cryptography by image filtering and resizing", Pers Ubiquit Comput, vol. 17, no. 5, pp. 843850, 2012.

[6] A. Kamboj and D. Gupta, "An Improved Halftone Visual Secret Sharing Scheme for Gray-Level Images Based on Error Diffusion in Forward and Backward Direction", 2015 Fifth International Conference on Advanced Computing \& Communication Technologies, 2015.

[7] E. Abdelsatir, S. Salahaldeen, H. Omar and A. Hashim, "A Novel $(K, N)$ Secret Sharing Scheme from Quadratic Residues for Grayscale Images", International Journal of Network Security \& Its Applications, vol. 6, no. 4, pp. 65-72, 2014.

[8] A. Mishra and A. Gupta, "Visual Cryptography For Gray Scale Image Using Block Replacement Half Toning Method", IEEE, vol. 6, no. 4, pp. 50-54, 2013.

[9] InKoo Kang, G. Arce and Heung-Kyu Lee, "Color Extended Visual Cryptography Using Error Diffusion", IEEE Transactions on Image Processing, vol. 20, no. 1, pp. 132-145, 2011.

[10] R. Jha, "Boolean Xor Based (K, N) Threshold Visual Cryptography for Grayscale Images", International Journal of Computer Science Issues (IJCSI), vol. 23, pp. 42-45, 2012.

[11] S. Katta, "Visual Secret Sharing Scheme using Grayscale Images", Cornell University Library, vol. 1, no. 1, p. 6, 2011. 